Los desequilibrios del sector externo y sus implicaciones para el esquema de dolarización en el Ecuador periodo 2010-2016

\title{
Los desequilibrios del sector externo y sus implicaciones para el esquema de dolarización en el Ecuador periodo 2010-2016
}
The imbalances of the external sector and its implications for the dollarization scheme in Ecuador period 2010-2016

\section{Os desequilíbrios do setor externo e suas implicações para o regime de dolarização no período do Equador 2010-2016}

Fredy G. Alvarado-Espinoza ${ }^{\mathrm{I}}$

freddyalvaradorp@hotmail.com

Mauro C. Tapia-Toral II mauro.tapiato@ug.edu.ec

Elaine R. Jijón-Gordillo III

elainejijon@hotmail.com

Recibido: 14 de enero de 2018 * Corregido: 19 de mayo de 2018 * Aceptado: 22 de junio de 2018

I. Docente de la Universidad de Guayaquil, Docente de la Universidad Agraria del Ecuador.

II. Docente de la Universidad de Guayaquil, Guayaquil, Ecuador.

III. Docente de la Universidad de Guayaquil, Guayaquil, Ecuador. 


\title{
Resumen
}

La presente investigación tuvo como objetivo analizar los desequilibrios del sector externo y sus implicaciones para el esquema de dolarización en el Ecuador dentro del período 2010 al 2017 a fin de orientar el análisis de los desequilibrios en la balanza de pagos, así como su impacto en el esquema monetario. Siguió una metodología de tipo descriptivo-explicativo-predictivo en un diseño no experimental-descriptivo- transaccional, en lo cual se analizaron los desequilibrios de la Balanza Comercial, el Endeudamiento Externo, el aporte de la Inversión Extranjera Directa, el aporte de las remesas de los emigrantes, el Déficit Fiscal y Externo y la volatilidad de los capitales de corto plazo. El corpus del estudio lo constituyeron las estadísticas nacionales consultadas desde el año 2010 al 2017, específicamente las del Boletín estadístico del BCE, del Banco Central e Información Estadística mensual. Los resultados permitieron concluir que el sector externo de la economía ecuatoriana presenta muchas debilidades que ponen en riesgo la dolarización. Una de estas es la situación de la balanza comercial, en especial la no petrolera que presenta déficits crecientes en todo el período analizado, lo que confirma la alta dependencia al petróleo.

Palabras clave: dolarización; balanza comercial; endeudamiento externo; déficit fiscal.

\begin{abstract}
The present study aimed to analyze the imbalances in the external sector and its implications for the scheme of dollarization in Ecuador within the period 2010 to 2017 in order to guide the analysis of the balance of payments imbalances, as well as its impact on the monetary scheme. Followed a methodology of descriptive -explicative type - predictive in a transactional not experimentaldescriptive-design, in which analyzed the imbalances in the trade balance, the external debt, the contribution of foreign direct investment the contribution of remittances from emigrants, the Fiscal Deficit and external and the volatility of short term capital. The corpus of the study constituted what national statistics consulted from 2010 to 2017, specifically those in the statistical bulletin of the ECB, the Central Bank and monthly statistical information. The results allowed concluding that the external sector of the Ecuadorian economy presents many weaknesses that put at risk the dollarization. One of these is the situation of the trade balance, especially not Oil Company presenting a growing deficit in all the analyzed period, thus confirming the high dependence on oil.
\end{abstract}

Keywords: dollarization; balance of trade; external indebtedness; fiscal deficit. 


\section{Resumo}

Esta pesquisa teve como objetivo analisar os desequilíbrios no setor externo e suas implicações para a dolarização no Equador, no período de 2010 a 2017 para orientar a análise dos desequilíbrios no balanço de pagamentos e seu impacto sobre esquema monetário. Metodologia seguida um preditivoexplicativo-descritivo num experimental não descritiva-transacional, em que os desequilíbrios são analisados balança comercial, empréstimos externos, a contribuição do IDE, a contribuição do design remessas emigrantes, o Déficit Fiscal e Externo ea volatilidade do capital de curto prazo. O corpus do estudo foi constituído pelas estatísticas nacionais consultadas de 2010 a 2017, especificamente as do Boletim Estatístico do BCE, do Banco Central e da Informação Estatística mensal. Os resultados permitiram concluir que o setor externo da economia equatoriana apresenta muitas fragilidades que colocam a dolarização em risco. Uma delas é a situação da balança comercial, especialmente a não petroleira, que mostra déficits crescentes ao longo do período analisado, o que confirma a alta dependência do petróleo.

Palavras chave: dolarização; saldo comercial; endividamento externo; déficit físcal.

\section{Introducción}

La Economía Ecuatoriana básicamente desde 1982 viene soportando una larga recesión económica, la misma que se extiende en la década de los noventa debido a la presencia de una serie de factores: El fenómeno del niño en 1998, la caída de los precios del petróleo en 1998, la crisis financiera que se agrava por el cierre de bancos y la transferencia al estado de más de la mitad de las instituciones financieras nacionales, desestabilización política e inestabilidad en general en los distintos mercados. La crisis se manifiesta en una vertiginosa expansión del desempleo abierto, el subempleo y la pobreza. El primero ascendió del 8\% en 1998 al 17\% en 1999, mientras la pobreza urbana pasó del 36\% al 65\% se produjo también una masiva migración internacional, al menos 700.000 ecuatorianos dejaron el país en 1999. Por otra parte, en este mismo año el PIB desciende a -6,3\%, la inflación alcanza el 60,7\% y la devaluación fue del $204 \%$. Frente a esta situación y ante la amenaza de la hiperinflación, el Gobierno del ex - presidente Mahuad, decreta en enero del año 2000, la dolarización oficial de la economía del Ecuador. 
La dolarización, plantea Benavides (1955), es un esquema monetario que elimina el riesgo de fluctuaciones en el tipo de cambio, los problemas de falta de concordancia entre activos en moneda local y deuda en dólares, la fuga de capitales y los ataques especulativos contra las reservas del Banco Central. Así mismo, la ausencia del instituto emisor implica que el gobierno no puede monetizar su déficit, lo que impone una restricción presupuestaria efectiva. Sin embargo, un proceso de esta naturaleza implica costos irrecuperables para el país, esto es el de abandonar la política cambiaria y monetaria, como instrumentos de estabilización económica. En efecto, explica Fernández (1995), se pierde capacidad discrecional para modificar el tipo de cambio ante perturbaciones externas e internas y la posibilidad de contar con política monetaria depende de acumular fondos de estabilización monetaria que permita a la autoridad económica influir en las tasas de interés y en el nivel de actividad económica. Por otra parte, indican Krugman y Odstfeld (1996), en dolarización el equilibrio interno pasa a depender directamente del equilibrio externo y lo fundamental es contar con un superávit permanente de balanza de pagos, para expandir la base monetaria en dólares y reactivar la economía. Una revisión general sobre los resultados de la dolarización diez y seis años después, nos dice: El PIB ha crecido en promedio al $3.7 \%$, debido a los altos precios del petróleo y las remesas de los emigrantes. La tasa de inflación anual ha pasado del 91\% en el 2000 al 3.6 en el 2016. Las tasas de interés activas se mantienen en promedio en 14\% entre 2000 y 2016 . El índice de desempleo ha pasado de $8.1 \%$ en el 2001, a 6.3\% en 2016. En esta perspectiva, la presente investigación está orientada al análisis de los desequilibrios de la balanza de pagos y su impacto en el esquema de dolarización del Ecuador.

\section{Desarrollo}

Históricamente, la economía ecuatoriana ha sido muy dependiente de pocos productos primarios de exportación; inicialmente del cacao, luego el banano y a partir de la década del setenta del petróleo. Esta dependencia no se ha modificado mayormente en la actualidad. En efecto, seguimos exportando en más del 70\% productos primarios en todo el período 2007 - 2016, lo que confirma que el grado de industrialización para exportación todavía es incipiente. Respecto a las importaciones, su estructura tampoco se ha modificado, las más significativas siguen siendo las de materias primas y bienes de capital para la industria. A este problema estructural, se agrega la aplicación del proceso de dolarización a partir del año 2000. 
Con este esquema monetario, el tipo de cambio se mantiene fijo en forma permanente y si consideramos los bajos niveles de productividad de los sectores productivo, el alto grado de apertura externa (70\% en 2015) y las devaluaciones de las monedas de los países socio comerciales, la situación se agrava para la balanza comercial, dado que el tipo de cambio real se aprecia, se abaratan las importaciones y se encarecen las exportaciones, lo que nos lleva a una permanente salida de divisas, que pone en riesgo el sistema de dolarización. La evidencia nos confirma que el saldo de la balanza comercial es negativo a partir del año 2009 hasta el 2015, de -233 a -2129 millones de dólares en el 2015. Para el año 2016 es positiva debido a la reducción de importaciones dada la caída del nivel de ingreso de este año por los efectos que genero el terremoto. Por otra parte, la alta dependencia de la balanza comercial al petróleo se evidencia cuando excluimos las exportaciones petroleras. En el periodo de análisis, la balanza comercial no petrolera es negativa, siendo el año más crítico en el 2012 que alcanzó 8350 millones de dólares. Esta situación pone en riesgo la estabilidad monetaria, más aún si consideramos que son factores estructurales tanto internos como externos que influyen en el saldo de la balanza comercial. Entre los factores internos, se pueden mencionar según Naranjo (2003), la reducida oferta exportable, el peso de las exportaciones no tradicionales, en el total es todavía muy bajo, producto precisamente de la baja productividad debido a limitaciones tecnológicas, financieras y de calidad del recurso humano. Además, tenemos la apreciación real del tipo de cambio, que nos conduce a un deterioro permanente de la balanza comercial, así también el sector industrial sigue dependiendo del exterior en materias primas y bienes de capital. Entre los factores externos, indica Porten (2002), está la permanente volatilidad de los precios de los productos de exportación, como el petróleo, banano, cacao, flores, sujeto a los ciclos económicos de los países desarrollados. En resumen, han sido los altos precios del petróleo que hemos tenido en la década de los 2000, los que han permitido sortear el déficit de la balanza comercial no petrolera y mantener la estabilidad monetaria. Sin embargo, su precio ha caído a partir del 2014, lo cual pone en riesgo la dolarización.

\section{Metodología}

La presente investigación constituyo una investigación de tipo descriptivo-explicativo-predictivo en un diseño no experimental-descriptivo- transaccional, en lo cual se analizaron los desequilibrios de la Balanza Comercial, el Endeudamiento Externo, el aporte de la Inversión Extranjera Directa, el aporte de las remesas de los emigrantes, el Déficit Fiscal y Externo y la volatilidad de los capitales de corto plazo. El corpus del estudio lo constituyeron las estadísticas nacionales consultadas desde el año 2010 
al 2017, específicamente las del Boletín estadístico del BCE, del Banco Central e Información Estadística mensual.

Análisis y discusión de los resultados.

El análisis de la información consultada y revisada, se presentan a continuación:

Tabla1. Balanza comercial del Ecuador en Millones de dólares

\begin{tabular}{|c|c|c|c|}
\hline \multirow{2}{*}{ AÑ OS } & \multicolumn{3}{|c|}{ BALANZA COMERCIAL } \\
\cline { 2 - 4 } & Total & Petroleras & $\begin{array}{c}\text { No } \\
\text { petroleras }\end{array}$ \\
\hline 2010 & -1979 & 5630 & -7609 \\
\hline 2011 & -829 & 7850 & -8687 \\
\hline 2012 & -440 & 8350 & -8769 \\
\hline 2013 & -1075 & 8237 & -9312 \\
\hline 2014 & -723 & 6917 & -4886 \\
\hline 2015 & -2129 & 2757 & -1722 \\
\hline 2016 & 1247 & 2969 & -7640 \\
\hline
\end{tabular}

Fuente: Banco Central.

Tabla 2. Deuda externa pública en relación al PIB

\begin{tabular}{|l|c|}
\hline Años & deuda/ PIB \\
\hline 1999 & $70,1 \%$ \\
\hline 2000 & $61.3 \%$ \\
\hline 2001 & $46.5 \%$ \\
\hline 2002 & $39.9 \%$ \\
\hline 2003 & $35.4 \%$ \\
\hline 2004 & $30.2 \%$ \\
\hline 2005 & $26,1 \%$ \\
\hline 2007 & $20.8 \%$ \\
\hline 2008 & $16.3 \%$ \\
\hline 2009 & $11.8 \%$ \\
\hline 2010 & $12.5 \%$ \\
\hline
\end{tabular}




\begin{tabular}{|l|c|}
\hline Años & deuda/ PIB \\
\hline 2011 & $12.7 \%$ \\
\hline 2012 & $12.4 \%$ \\
\hline 2013 & $13.6 \%$ \\
\hline 2014 & $17.2 \%$ \\
\hline 2015 & $20.2 \%$ \\
\hline 2016 & $28.7 \%$ \\
\hline 2017 & $39.6 \%$ \\
\hline
\end{tabular}

Fuente: Banco Central

La deuda externa, tal como la presenta el banco central del Ecuador (2010-2018), se convirtió en la década de los ochenta y los noventa en el principal problema para la economía ecuatoriana. El endeudamiento crece desde mediados de la década de los setenta debido a factores internos y externos. Entre los primeros está el modelo de industrialización sustitutiva que requirió de grandes volúmenes de divisas por su alto grado de dependencia con el exterior, en la compra de materias primas y bienes de capital. La expansión del sector público acorde con el modelo de desarrollo, conduce a mantener altos niveles de crecimiento del gasto público. La producción y exportación de petróleo nos convierte en atractivos sujetos de crédito. El mantenimiento de un sistema de tipo de cambio fijo (25 sucres/dólares) de 1970 - 1981 con bajo riesgo cambiario, e incentiva el endeudamiento público y privado. Entre los factores externos está la sobreabundancia de créditos de la banca internacional, cuyos recursos provienen de los países exportadores del medio oriente, que se favorecieron con los altos precios del petróleo. El Alza de las tasas de interés en especial a partir de la crisis financiera mundial de 1982 - 1983. Como resultado de estos factores, la deuda creció afectando básicamente al presupuesto general del Estado, tanto el gasto corriente y de capital. En promedio, la deuda representa más del $50 \%$ del gasto público, cerca del 100\% del PIB desde mediados de los ochenta y durante los noventa. Así mismo hemos tenido que declarar dos veces moratoria en los años de 1987 y 1999.

Respecto a la evolución de la deuda externa, que presenta el banco central del Ecuador (2010-2018), la tendencia es que la deuda pública en relación al PIB pasa de un nivel de $61.3 \%$ en el año 2000 a $20.8 \%$ en el 2007 y $11.8 \%$ en el 2009 , esta reducción importante del peso de la deuda se explica por el incremento de la tasa de crecimiento del PIB y la renegociación que se alcanzó en el año 2008 .A 
partir de este año la deuda mantiene una tendencia creciente hasta alcanzar $39.65 \%$ en el año 2017 , situación que se agrava por la caída de los precios del petróleo y el terremoto del año 2016.

Tabla 3. Inversión extranjera directa en millones de dólares durante el período 2002 - 2017

\begin{tabular}{|ccccccccccccccccc|}
\hline AÑO & 200 & 200 & 200 & 200 & 200 & 200 & 200 & 200 & 201 & 201 & 201 & 201 & 201 & 201 & 201 & 201 \\
S & 2 & 3 & 4 & 5 & 6 & 7 & 8 & 9 & 0 & 1 & 2 & 3 & 4 & 5 & 6 & 7 \\
& & & & & & & & & & & & & & & & \\
\hline IED & 783 & 872 & 837 & 493 & 271 & 193 & 996 & 308 & 165 & 644 & 567 & 727 & 772 & 132 & 755 & 605 \\
\end{tabular}

\section{Fuente: Banco Central}

Por inversión extranjera directa se entienden los flujos internacionales de capital en los que una empresa de un país crea o amplía una filial en otro país. Las causas que originan las inversiones directas en el exterior es la de lograr rendimientos más altos y diversificar los riesgos. Estas inversiones se realizan a través de las empresas multinacionales, que es un medio de extensión del control sobre las actividades que tiene lugar en 2 o más países. El objetivo principal es aprovechar las economías de localización, es decir concentrar las actividades en países que se cuente con abundancia de recursos humanos y naturales para reducir costos de producción. La inversión extranjera directa es de mediano y largo plazo y se orienta en los distintos sectores productivos de la economía; su aporte es fundamental para dinamizar la producción y el empleo. Uno de los beneficios que traería la dolarización en el Ecuador, es el aporte de esta inversión, dada la eliminación de la incertidumbre en el tipo de cambio y el hecho de realizar inversiones en dólares, sin embargo, la realidad es distinta porque ha sido insignificante y además ha ido disminuyendo de 783 millones de dólares en el 2002 a 606.43 millones de dólares en el 2017.

En promedio en el periodo 2002 al 2017 la inversión extranjera directa es de 648 millones de dólares. Lo más preocupante es que más del 90\% es inversión en el área petrolera. El hecho que su aporte a la economía nacional es muy baja, a pesar del esquema monetario adoptado confirma que existen otros elementos que los inversionistas consideran en el momento de realizar inversiones como: el tamaño del mercado, el poder adquisitivo de los consumidores, que depende de las expectativas de crecimiento económico, a estos factores se agregan el propio sistema económico y el régimen de derechos de 
propiedad, la calidad de las instituciones democráticas, la seguridad jurídica, la estabilidad política. En relación a esta última, el riesgo político ha sido definido como la probabilidad de que las fuerzas políticas causen cambios drásticos en el medio empresarial. En este escenario, la inversión no aporta realmente a la estabilidad monetaria y se requiere cambios drásticos para lograr sostener el esquema de dolarización.

\section{La volatibilidad de los capitales de corto plazo.}

Al igual que en el caso de la inversión extranjera directa, el motivo fundamental para que se lleven a cabo inversiones de cartera o portafolio es obtener mayores ganancias. Por esta razón, los residentes de un país compran bonos emitidos por otro país, si los rendimientos de dichos bonos son más elevados en este. Las variables que influyen en esta rentabilidad, considerando un país con moneda propia, son:

$\mathbf{i}=$ tasa de interés local $\quad \mathbf{i x}=$ tasa de interés extranjero

$\mathbf{T C e}=$ tipo de cambio esperado $\quad \mathbf{T C}=$ tipo de cambio de hoy

$\mathbf{P}=$ prima por riesgo

Supongamos que el tipo de cambio es flexible, para atraer capitales se requiere:

$\mathbf{i}>\mathbf{i x}+(\mathbf{T C e}-\mathbf{T C})+\mathbf{P} ; \mathbf{i}=$ rentabilidad en moneda local

TC

$\mathbf{i X}+(\mathbf{T C e}-\mathbf{T C})+\mathbf{P}=$ rentabilidad en moneda extranjera

TC

\section{Con tipo de cambio fijo:}

$\mathbf{T C e}=\mathbf{T C}$; por lo tanto, la tasa de depreciación es cero, y para atraer capitales: $\mathbf{i}>\mathbf{i x}+\mathbf{P}$

Tabla 4. Inversión de cartera en millones de dólares durante el período 2000 - 2009

\begin{tabular}{|c|c|c|c|c|c|c|c|c|c|c|c|c|c|c|c|c|}
\hline AÑOS & 2002 & 2003 & 2004 & 2005 & 2006 & 2007 & 2008 & 2009 & 2010 & 2011 & 2012 & 2013 & 2014 & 2015 & 2016 & 2017 \\
\hline Inversión & & & & & & & & & & & & & & & & \\
\hline $\begin{array}{c}\text { de } \\
\text { Cartera }\end{array}$ & 0 & -304 & -190 & 366 & -1384 & -118 & 213 & -3141 & -731 & 41 & 66 & -909 & 1500 & 1473 & 2200 & 1622 \\
\hline
\end{tabular}

Fuente: Boletín estadístico del BCE 
El problema de los regímenes de tipos de cambio fijo es que necesitan de ciertas precondiciones para sostenerse por lo que resulta mucho más efectivo para atraer capitales a los regímenes con tipo de cambio extremo; como es el caso del sistema de dolarización. En efecto, bajo este esquema desaparece el riesgo de devaluación y los capitales deben ingresar con más facilidad. Esta es una coyuntura que no ha sido aprovechada o que Ecuador no se beneficie de una de las mayores ventajas de la dolarización, es decir no hay la suficiente confianza en los compradores de títulos públicos, ni en las entidades de crédito alternativamente, en nuestro país, el peso de la deuda es tan significativo que hemos tenido que endeudarnos para cumplir con los compromisos externos, a esto se agrega la indisciplina fiscal y el aumento en la tasa de inflación. Por otra parte, si bien estos capitales son especulativos y no es recomendable a largo plazo su permanencia, sin embargo, en dolarización coadyuvan a disminuir la tasa de interés, contribuyendo a la estabilidad monetaria.

Si tomamos como referencia la inversión de cartera en el período analizado en casi todos los años, su saldo es cero o negativo, a excepción del año , 2005,2008, 2011, 2012,y del periodo 2014 - 2017, debido a la emisión de títulos de deuda para financiar el gasto público a raíz de la caída de los precios del petróleo y el problema del terremoto del año 2016 y 2009, La situación se agrava por los altos montos por el pago de intereses de la deuda medida a través de la renta de inversión de cartera negativa.

Tabla 5. Déficit o superávit fiscal y el saldo balanza pagos y cuenta corriente en millones de dólares durante el período $2010-2017$.

\begin{tabular}{|ccccccccc|}
\hline Años & 2010 & 2011 & 2012 & 2013 & 2014 & 2015 & 2016 & 2017 \\
\hline Déficit/Superávit Fiscal & 944 & 100 & 824 & 4347 & 5314 & 5940 & 7313 & 4653 \\
\hline $\begin{array}{l}\text { Déficit/Superávit Balanza } \\
\text { Pagos y Cuenta Corriente. }\end{array}$ & 1606 & 260 & 164 & 983 & 522 & 2226 & 1316 & 348 \\
\hline
\end{tabular}

Fuente: Boletín estadístico del BCE

Existe una estrecha relación entre el déficit o superávit fiscal y el saldo de la Balanza de Pagos en cuenta corriente. Básicamente un déficit fiscal nos conduce a un déficit de la balanza de pagos en cuenta corriente; así como un superávit del sector público es sinónimo de superávit en cuenta corriente. La evidencia empírica nos revela que, para el caso del Ecuador, existe una relación muy 
estrecha entre estas variables. El sector público presenta déficit fiscal en todo el periodo de análisis, por su parte la cuenta corriente de la balanza de pagos, el déficit se presenta desde el año 2010 a 2017 , Lo que confirma la hipótesis del déficit fiscal influyen en el sector externo. Específicamente el exceso de gasto público aumenta la demanda de importaciones, lo cual nos conduce a un déficit comercial. La situación se agrava en la medida que en los últimos años las cuentas fiscales mantienen un abultado déficit fiscal; como consecuencia del crecimiento del gasto público corriente de 20430 millones de dólares en el 2010 a 28991 en el 2017 y. El crecimiento del gasto público genera el problema de la sostenibilidad con los ingresos. Los mecanismos utilizados han sido básicamente el aumento de impuestos, lo cual a su vez genera mayores distorsiones en la economía nacional, en especial para la producción y el empleo.

Tabla 6. Remesas de los emigrantes en millones de dólares durante el período 2010 -2016

\begin{tabular}{|cccccccccc|}
\hline Años & 2000 & 2007 & 2010 & 2011 & 2012 & 2013 & 2014 & 2015 & 2016 \\
\hline Remesas & 1316 & 3086 & 2591 & 2672 & 2466 & 2449 & 2461 & 2377 & 2602 \\
\hline
\end{tabular}

Fuente: Boletín estadístico del BCE

Las remesas enviadas por los emigrantes han sido un puntal para la sostenibilidad de la dolarización en el Ecuador, convirtiéndose después del petróleo en el principal rubro de ingreso de divisas. En efecto, a raíz de la crisis económica de finales de los noventa, se produce un éxodo masivo de ecuatorianos hacia Europa y los Estados Unidos, generándose una nueva fuente de ingresos, cuyo monto aumenta de 1316 millones de dólares en el año 2000 a 3087 millones en el 2007, para el año 2010 disminuyen a 2591, siguen bajando a 2377 millones en el 2015, y aumentan ligeramente a 2602 en el 2016. Las remesas recibidas se orientan básicamente al consumo y la inversión, lo cual genera un efecto multiplicador sobre el nivel de la actividad económica. En este escenario, la expectativa es que las remesas se desaceleren en los próximos años, lo que contribuirá a la inestabilidad y al colapso de la dolarización. 


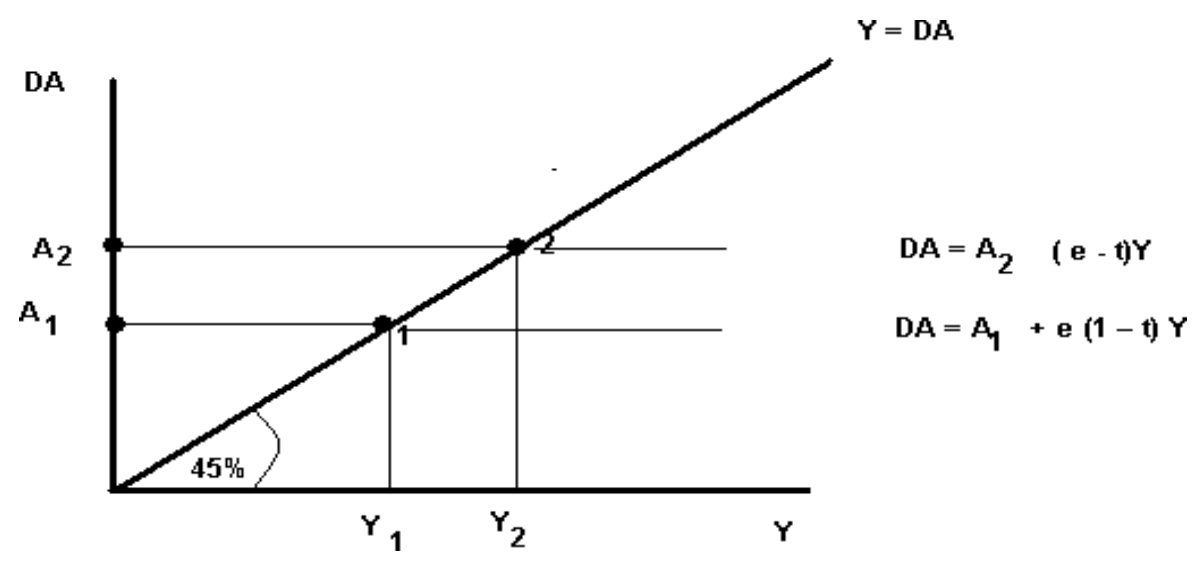
$\mathbf{A}$ = gasto agregado
$\mathbf{E}=$ consumo autónomo
$\mathbf{I}=$ inversión autónoma
$\mathbf{c}=$ Pmg. Consumir
$\mathbf{t}=$ impuesto
$\mathbf{P}=$ precios constantes

Grafico 1. Comportamiento de las remesas

Autor. Elaboración propia.

Como ilustramos en el gráfico 1, el aumento de las remesas permite aumentar el ingreso y/o la producción, sin embargo, parte de esta renta se orienta al exterior, a través de mayores importaciones, contribuyendo al déficit de la balanza comercial. Por otra parte, la tendencia en los últimos años ha sido que las remesas disminuyen como consecuencia de varios factores:

La crisis económica en Estados Unidos, del 2007-2008, significó pérdidas de empleo para los ecuatorianos.

La crisis económica de Europa en el 2010, significó mayores ajustes, en especial fiscal y monetario, que conducen a reducir el gasto público y aumento de las tasas interés, con lo cual la actividad económica se deprime y aumenta el desempleo.

Los países europeos han endurecido las Leyes inmigratorias y los flujos de remesas deben disminuir en los próximos años.

La depreciación del euro significa menores ingresos en dólares para la economía, tanto por el lado de las exportaciones, como de las remesas recibidas.

El tipo de cambio real y la pérdida de competitividad 
El tipo de cambio real es el precio relativo entre dos bienes o dos canastas de bienes. El bien local vers. El bien extranjero, este precio es muy importante para el equilibrio de la economía porque mide la competitividad externa del país en lo que respecta a los precios y responde a la pregunta ¿La producción nacional es muy cara o barata frente a la producción de otros países? Para calcular el tipo de cambio real necesitamos conocer los precios nacionales y extranjeros de los bienes y el tipo de cambio nominal.

\section{La fórmula es:}

$\mathbf{T C R}=\mathbf{E P x} ;$ donde

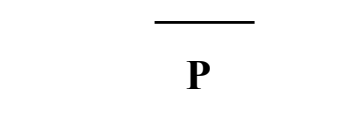

$\mathbf{P}=$ precio local $\quad \mathbf{P x}=$ precio externo

$\mathbf{E}=$ tipo de cambio nominal $\mathbf{T C R}=$ tipo de cambio real

Supongamos que $1 \mathrm{qq}$ de arroz de Ecuador cuesta 20 dólares, el mismo qq de arroz en Colombia cuesta 200 pesos, si el tipo de cambio nominal es 10 pesos/dólar ó 0.1 dólar/peso. El tipo de cambio real es:

$\mathbf{T C R}=0.1 \underline{\mathrm{dol} / \mathrm{peso} \times 200 \text { pesos }}$

20 dólares

TCR $=1$, lo que significa que 1 qq de arroz en Ecuador $=1$ qq arroz de Colombia, es decir, la competitividad es la misma para los dos países; ahora, si la inflación en el Ecuador aumenta a un 25\% y Colombia devalúa la moneda en un $30 \%$. El tipo de cambio real es:

$$
\mathbf{T C R}=0.077 \underline{\mathrm{dol} / \mathrm{peso} \times 200 \mathrm{pesos}}=0.62
$$

\section{5 dól.}

Lo que significa que 0.62 qq de arroz de Ecuador, es igual a 1qq de arroz de Colombia, esta situación hace que el producto local se encarezca frente al extranjero y la demanda se desplaza de bienes nacionales a bienes importados. Cuando se produce una caída del tipo de cambio real, expresan Sachs y Larraín (2014), decimos que existe una apreciación real del tipo de cambio. Esto es precisamente 
lo que ha ocurrido en el período 2010 - 2016, lo que se explica por las constantes devaluaciones de las monedas de los socios comerciales del Ecuador como observamos en el siguiente cuadro.

Tabla 7. Tipo de cambio en el mundo, frente al dólar

\begin{tabular}{|l|r|r|r|r|r|r|r|}
\hline PAÍSES & $\mathbf{2 0 1 0}$ & $\mathbf{2 0 1 1}$ & $\mathbf{2 0 1 2}$ & $\mathbf{2 0 1 3}$ & $\mathbf{2 0 1 4}$ & $\mathbf{2 0 1 5}$ & $\mathbf{2 0 1 6}$ \\
\hline Ecuador & 25000 & 25000 & 25000 & 25000 & 25000 & 25000 & 25000 \\
\hline Colombia & 1835 & 1939 & 1760 & 1925 & 2388 & 3169 & 2996 \\
\hline Perú (S/D.) & 2.8 & 2.68 & 2.55 & 2.79 & 2.98 & 3.41 & 3.34 \\
\hline Venezuela & 3.45 & 4.29 & 6.28 & 6.28 & 9.97 & 9.975 & 9.975 \\
\hline Brasil & 1.66 & 1.86 & 2.04 & 2.35 & 2.66 & 3.99 & 3.25 \\
\hline Argentina & 3.97 & 4.30 & 4.91 & 6.51 & 8.54 & 12.97 & 15.67 \\
\hline
\end{tabular}

Fuente: Boletín estadístico del BCE,

Como resultado de lo anterior el tipo de cambio bilateral real del Ecuador frente a otros países se deteriora, lo cual unido a factores reales, le resta competitividad al país en el mercado internacional.

Tabla 8. Índice del tipo de cambio real en Base 1994 = 100 durante el periodo 2010 - 2016

\begin{tabular}{|crrrrrrr|}
\hline Años & 2010 & 2011 & 2012 & 2013 & 2014 & 2015 & 2016 \\
\hline $\begin{array}{c}\text { Efectivo } \\
\text { real }\end{array}$ & 98 & 96 & 94 & 103 & 100 & 88 & 86 \\
\hline
\end{tabular}

Fuente: Información Estadística mensual.

En relación al año 2010 el tipo de cambio real del Ecuador frente a otros países que mantenemos fuertes lazos comerciales se aprecia lo cual ha contribuido al déficit de la balanza comercial. Si bien en el año 2013 el tipo de cambio real se deprecia, a partir de este año mantiene una tendencia a la baja. La situación es más grave si consideramos el abultado déficit de la balanza comercial no petrolera, tal como indicamos anteriormente. 


\section{Conclusiones}

El sector externo de la economía ecuatoriana presenta muchas debilidades que ponen en riesgo la dolarización. Una de estas es la situación de la balanza comercial, en especial la no petrolera que presenta déficit creciente en todo el período analizado, lo que confirma la alta dependencia al petróleo. Respecto a la deuda externa, si bien su peso en relación al PIB ha disminuido de $71.1 \%$ en 2000 a $14.4 \%$ en el 2009 si embargo para el 2017 aumenta al 39\% lo que pone en riesgo al esquema monetario. Otra de las debilidades encontradas es el poco aporte de la inversión extranjera directa a la actividad económica, de 720 millones de dólares en el 2000, disminuye a 625 millones en el 2009 y 605 en el 2017, la misma situación se observa en los capitales de corto plazo, a pesar de contar con el dólar y sin riesgo cambiario. Respecto al déficit fiscal, la evidencia para el Ecuador nos revela que existe una relación muy estrecha con el déficit de Balanza de Pagos. En el gobierno tenemos déficit en todo el período de análisis, igual situación tenemos en la balanza en cuenta corriente.

El aporte de las remesas ha sido vital para el sostenimiento de la dolarización, al pasar de 1316 millones de dólares en 2000 a 2495 millones en el 2009 y 2602 en el 2016; sin embargo, la crisis mundial está provocando una disminución de estos ingresos. En dolarización, con tipo de cambio fijo extremo, el tipo de cambio real se aprecia con lo cual disminuyen las exportaciones y aumentan las importaciones, a esto se suma las devaluaciones de las monedas de los países socio comerciales, con lo cual el déficit comercial aumenta. Para sostener la dolarización, es fundamental aumentar el nivel de competitividad, a través de una serie de mecanismos como la inversión de capital humano, mayor infraestructura física y de servicios básicos, reformas financieras, para que la banca canalice los fondos de ahorro a la inversión, aumentar el gasto en investigación y desarrollo, estabilidad económica y política, apertura económica, control de monopolio. En esta misma dirección, la integración financiera con la banca internacional es una tarea fundamental, se requiere incentivar la apertura de instituciones financieras del exterior para propiciar mayor competencia, menores tasas de interés y contar con prestamistas de última instancia. Por su parte es necesario reducir el gasto público, o que éste crezca en base a fuentes permanentes de ingresos y no de recursos extraordinarios, de la experiencia Argentina es el crecimiento del gasto y la deuda la que ocasionó el colapso de la convertibilidad.

La reforma comercial debe incluir los acuerdos de libre comercio con EEUU y Europa; se debe contar con un buen equipo de negociadores para no afectar a los sectores productivos vulnerables. 


\section{Referencias Bibliográficas}

Banco Central del Ecuador. (2010 - 2018). Boletines Estadísticos.

Benavides, J (1995). (3ra. Edición) España. Editorial McGraw - Hill. - año 1995.

Fernández A. (1995). Política Económica. España. Editorial McGraw - Hill.

Krugman, P y Obstfeld M (1996). Economía Internacional: Teoría y Política. (3ra. Edición) España.

Editorial McGraw - Hill.

Naranjo Marco. (2003). Regímenes Monetarios y Dolarización. Banco Central del Ecuador.

Porter Michael. (2002). Ventaja Competitiva. México. Editorial CECSA.

Sachs - Larraín (2014). Macroeconomía en la Economía. (2da. Edición) Argentina.

Global Editorial Prentice Hall. 